\title{
The Calculation Method of One-dimensional Magneto Rheological Elastomer Problem
}

\author{
Zhenguo Tian ${ }^{1,}$, , Jinkui Liang ${ }^{1, b}$ \\ ${ }^{1}$ Key Laboratory of Mechanical Reliability for Heavy Equipments and Large Structures of Hebei \\ Province, Yanshan University, Qinhuangdao 066004, China \\ atianzhenguo1@163.coml, b1475828755@qq.com
}

\begin{abstract}
Keywords: magnetorheological elastomer component; coupled field; nonlinear; numerical methods; stress.

Abstract. When the magnetorheological member is in the strong magnetic field and the mechanical field, it will produce a magnetic induction effect which affects the variation of the modulus of elasticity. The variation of modulus has an effect on the deformation of the component. And the deformation also affects the magnetic field distribution. So it is the coupling effect, and the constitutive relation is nonlinear. Based on the motion equations, electrodynamics equations and the constitutive relation, the magnetorheological elastomer component basic equations in the mechanical field and the magnetic field are obtained. Considering the property of real time change of material properties and the norm load in plane, using DOM method to get the numerical solution of the magnetorheological elastomer. Furthermore, taking the annular plate as an example, deformation and the stress distribution of axisymmetric magnetorheologi- cal elastomer component in coupled field is calculated.
\end{abstract}

\section{Introduction}

Magnetorheological elastomer, as an intelligent material, has been applied in Engineering[1,2], the research on the properties of magnetorheological elastomer materials is also carried out. Dorfmann et al., respectively, using the invariant theory and the strain energy function to analyze the mechanical properties of the magnetorheological elastomers in plate shear and axial shear[3]. However, it is rare to analyze the mechanical properties of magnetorheological elastomer as a structural component under the action of mechanical and electromagnetic fields. Magnetorheological elastomer research focuses on material preparation, mechanical performance test and establish constitutive relations[4]. Magneto-elastic coupling problem object before mainly copper, iron, aluminum and other metal materials[5,6]. Magnetorheological elastomer made of the test pieces into the electromagnetic field, the magnetic particles in a changing magnetic field within the magnetorheological elastomer sheet will produce a magnetic induction effect, thereby affecting the variation of the modulus of elasticity of the plate, further impact of changes in the modulus of elasticitythe deformation of the sheet, and the deformation in turn affect the magnetic field distribution, which formed the coupling relationship.

In this paper, according to the motion equation and the electrodynamics equations and magnetic fluid constitutive relation, A set of partial differential equations with 8 unknown quantities describing the mechanical properties of magneto rheological elastomers are established, On the basis of DOM[7], and the characteristics of elastic modulus of magnetorheological elastomers in real time, the numerical calculation method of one dimensional magneto rheological elastomer under the action of electromagnetic field and mechanical field is obtained, the corresponding calculation flow chart is given, the program is compiled by using Fortran software. As an example, the deformation and stress of the annular magneto rheological elastomer thin plate under the action of magnetic field and mechanical load are calculated.

\section{Basic equations of magneto rheological elastomers}

In the dipole model [8], the shear modulus caused by the magnetic field 


$$
G \approx \frac{\phi J_{p}^{2}}{2 \mu_{2} \mu_{0} h_{0}^{3}}
$$

Where $\phi$ is a particle volume fraction, $J_{P}=\frac{x_{m} B_{3}}{1+x_{m}}$ is the polarization of the particles, $\mu_{0}$ is the permeability of vacuum, $\mu_{2}$ is the magnetic permeability of the magnetic medium, $h_{0}$ is a gap between the particles of directions, $x_{m}$ is the magnetic susceptibility. The elastic modulus of magnetorheological elastomer material can be obtained from the magnetic induction strength and the magnetic shear modulus

$$
E=2(1+v) \frac{\phi x_{m}^{2} B_{3}^{2}}{2 \mu_{2} \mu_{0}\left(1+x_{m}\right)^{2} h_{0}^{3}}=\frac{\phi(1+v) B_{3}^{2} x_{m}^{2}}{\mu_{2} \mu_{0}\left(1+x_{m}\right)^{2} h_{0}^{3}}
$$

Where $v$ is the Poisson's ratio, $B_{3}$ is the normal magnetic induction intensity.

According to the motion equations, electrodynamics equations, elastic relations and geometry relations, dynamic equations of annular plate is available ${ }^{[7]}$

$$
\begin{gathered}
\frac{\partial u}{\partial \alpha}=\frac{A(1-v)}{E h} N_{1}-A\left(\frac{1}{R_{1}}+\frac{v}{R_{2}}\right) w-\frac{v}{B} \frac{\partial B}{\partial \alpha} u-0.5 A \theta_{1}^{2} \\
\frac{\partial w}{\partial \alpha}=-A \theta_{1}+\frac{A}{R_{1}} u \\
\frac{\partial \theta_{1}}{\partial \alpha}=\frac{12 A\left(1-v^{2}\right)}{E h^{3}} M_{1}-\frac{v}{B} \frac{\partial B}{\partial \alpha} \theta_{1} \\
\frac{\partial N_{1}}{\partial \alpha}=\frac{1}{B} \frac{\partial B}{\partial \alpha}\left[(v-1) N_{1}+E h\left(\frac{1}{A B} \frac{\partial B}{\partial \alpha} u+\frac{w}{R_{2}}\right)\right]-\frac{A}{R_{1}} Q_{1}-A\left(P_{1}+n_{1}+\rho f_{1}^{\phi}\right)+A \rho h \frac{\partial^{2} u}{\partial t^{2}} \\
\frac{\partial Q_{1}}{\partial \alpha}=-\frac{1}{B} \frac{\partial B}{\partial \alpha} Q_{1}+A\left[\left(\frac{1}{R_{1}}+\frac{v}{R_{2}}\right) N_{1}+\frac{E h}{R_{2}}\left(\frac{1}{A B} \frac{\partial B}{\partial \alpha} u+\frac{w}{R_{2}}\right)\right]-A\left(P_{3}+n_{3}+\rho f_{3}^{\phi}\right)+A \rho h \frac{\partial^{2} w}{\partial t^{2}} \\
\frac{\partial B}{B \alpha}\left[(v-1) M_{1}+\frac{E h^{3}}{12 A B} \frac{\partial B}{\partial \alpha} \theta_{1}\right]+A\left[N_{1}-\frac{1}{R_{2}}\left(v M_{1}+\frac{E h^{3}}{12 A B} \frac{\partial B}{\partial \alpha} \theta_{1}\right)\right] \theta_{1}+ \\
\frac{\partial B_{3}}{\partial \alpha}=-A \sigma \mu\left[E_{2}+\frac{1}{2} \frac{\partial w}{\partial t}\left(B_{1}^{+}+B_{1}^{-}\right)-\frac{\partial u}{\partial t} B_{3}\right]+\frac{A}{B} \frac{B_{1}^{+}-B_{1}^{-}}{h} \\
\frac{\partial E_{2}}{\partial \alpha}=-A \frac{\partial B_{3}}{\partial t}-\frac{1}{B} \frac{\partial B}{\partial \alpha} E_{2}
\end{gathered}
$$

In the formula, $u, w, \theta_{1}$ respectively displacements, deflections and rotations in the corresponding direction of the thin plate; $M_{1}, N_{1}, Q_{1}$ respectively, the corresponding direction of the sheet bending moment, axial force and shear; $P_{1}, P_{2}, P_{3}$ are respectively the mechanical surface forces in the respective directions; $B_{1}^{+}, B_{1}^{-}$respectively to the radial magnetic field in the thin plate, the lower surface of the component; $E_{2}$ is the circumferential electric field strength; $t$ is the time; $\sigma$ is the dielectric conductivity; $h$ is the plate thickness; $\rho$ is the mass density of the medium; $E$ is the modulus of elasticity; $v$ is the Poisson ratio; $\mu$ is the magnetic permeability; $A, B$ are the Lame coefficients; $n_{1}, n_{2}, n_{3}$ are respectively electromagnetic load concentration; $\rho f_{1}^{\phi}, \rho f_{3}^{\phi}$ respectively to the radial and Lorenz force, in this paper, only the $n_{3}$ is considered, the magnetic force may be equivalent to the equivalent surface force acting on the middle plane can be calculated as 


$$
n_{3}=2 \pi \mu_{1} R_{0}^{3} K \nabla H_{3}^{2}
$$

Where $K=\frac{\mu_{2}-\mu_{1}}{\mu_{2}+2 \mu_{1}}, \mu_{1}$ is the permeability of colloidal particles, $R_{0}$ is the radius of colloidal particles.

$$
\nabla H_{3}^{2}=\nabla\left(\frac{B_{3}^{2}}{\mu_{0}^{2}\left(1+x_{m}\right)^{2}}\right)=\frac{2 B_{3}}{\mu_{0}^{2}\left(1+x_{m}\right)^{2}} \frac{\partial B_{3}}{\partial \alpha}
$$

So

$$
n_{3}=2 \pi \mu_{1} R_{0}^{3} \frac{\mu_{2}-\mu_{1}}{\mu_{2}+2 \mu_{1}} \frac{2 B_{3}}{\mu_{0}^{2}\left(1+x_{m}\right)^{2}} \frac{\partial B_{3}}{\partial \alpha}=\frac{4 \pi \mu_{1} R_{0}^{3} B_{3}\left(\mu_{2}-\mu_{1}\right)}{\mu_{0}^{2}\left(\mu_{2}+2 \mu_{1}\right)\left(1+x_{m}\right)^{2}} \frac{\partial B_{3}}{\partial \alpha}
$$

\section{Linearization of basic equations}

Eq.(3) expresses a nonlinear differential equation set. In order to solve it, it could be rewritten into the following form for convenience of description

$$
\frac{\mathrm{d} \boldsymbol{f}}{\mathrm{d} \alpha}=\boldsymbol{F}(\alpha, \boldsymbol{f}) \quad(a \leq \alpha \leq b)
$$

Boundary condition:

$$
\begin{aligned}
& D_{1} \boldsymbol{f}(a)=\boldsymbol{d}_{1} \\
& D_{2} \boldsymbol{f}(b)=\boldsymbol{d}_{2}
\end{aligned}
$$

Where $\boldsymbol{F}$ is an n-dimensional vector $\boldsymbol{f}=\left\{f_{1}, f_{2}, \cdots, f_{n}\right\}^{T}$ is an n-dimensional longitudinal vector, $\boldsymbol{D}_{1}, \boldsymbol{D}_{2}$ are orthogonal matrixes with the order of $k \times n$ and $(n-k) \times n$ respectively. $\boldsymbol{d}_{1}, \boldsymbol{d}_{2}$ are given vectors. The approximate solution of the boundary value problem Eq. (6) and Eq. (7) is $\boldsymbol{f}^{(k)}(\alpha)$, at this point, the exact solution $\boldsymbol{f}_{*}^{(k)}(\alpha)$ can be recorded in the following form

$$
\boldsymbol{f}_{*}^{(k)}(\alpha)=\boldsymbol{f}^{(k)}(\alpha)+\Delta \boldsymbol{f}_{*}^{(k)}(\alpha)
$$

Considering the boundary conditions, the quasi linear iterative equation can be obtained

$$
\begin{gathered}
\frac{\mathrm{d} \boldsymbol{f}^{(k+1)}}{\mathrm{d} x}=\boldsymbol{F}\left(\alpha, \boldsymbol{f}^{(k)}\right)+\boldsymbol{\Gamma}\left(\alpha, \boldsymbol{f}^{(k)}\right)\left(\boldsymbol{f}^{(k+1)}-\boldsymbol{f}^{(k)}\right) \\
D_{1} \boldsymbol{f}^{(k+1)}(a)=\boldsymbol{d}_{1} \quad ; \quad D_{2} \boldsymbol{f}^{(k+1)}(b)=\boldsymbol{d}_{2} \quad(k=0,1,2 \cdots)
\end{gathered}
$$

Where $\boldsymbol{\Gamma}\left(\alpha, \boldsymbol{f}^{(k)}\right)$ is the Jacobi matrix of the right part of the system. So far, it can be solved by the discrete orthogonal method, the iterative process described in the Eq.(9) is established at each discrete point, according to the boundary conditions, the numerical solution of the problem can be obtained.

\section{Program design of orthogonal discrete method}

Fig. 1 is a flow chart for solving the problem of Magnetorheological Elastomers. The $t_{N}$ is the end time of the calculation, $\Delta t$ is a time interval. The basic equation of magneto rheological elastomer Eq.(3) is a system of nonlinear partial differential equations. At each time point, a series of equations are linearized, a series of ordinary differential equations are obtained, then, by introducing the boundary conditions, the Schmidt method is used to orthogonal the process variables, to solve a series of linear ordinary differential equations with Adams method ${ }^{[9]}$, calculate each point in turn, and save the process variables and the corresponding data, then, according to the boundary conditions of the right side, the process coefficients are obtained, the function value of each discrete point is obtained, Thus, the function value of each time point can be obtained. It should be noted that the choice of the specific algorithm will affect the convergence interval, thereby affecting the stability of the calculation and the accuracy of the calculation. The response of the magnetorheological elastomers to the magnetic field is much larger than that of the conventional materials, it is characterized by large change of elastic 
modulus, large displacement value, fast change speed and so on, therefore, it is necessary to take the time difference interval value is small enough, and pay attention to the real-time relationship between the variables of each subroutine.

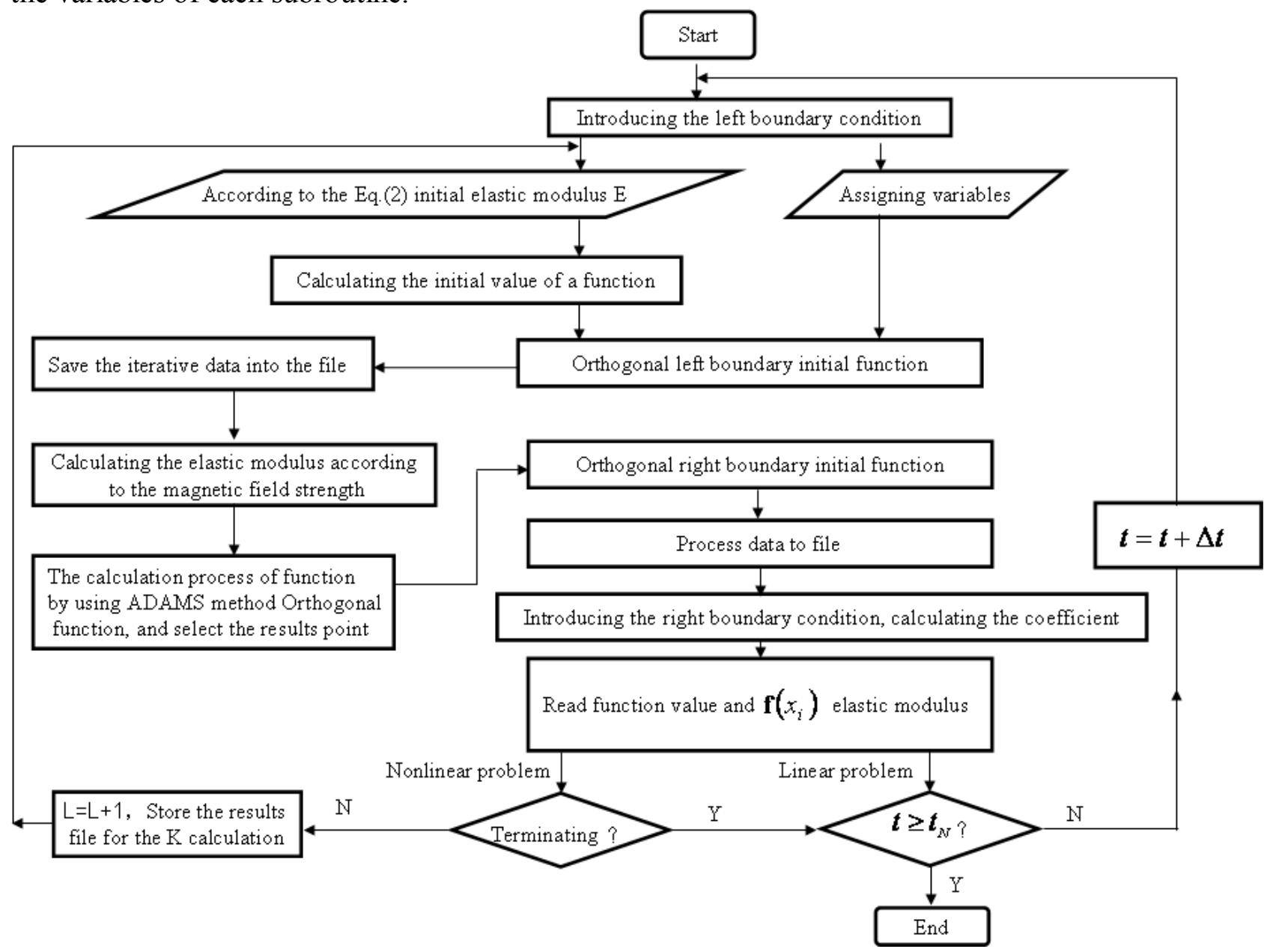

Fig. 1 The flow chart of DOM

\section{Example analysis}

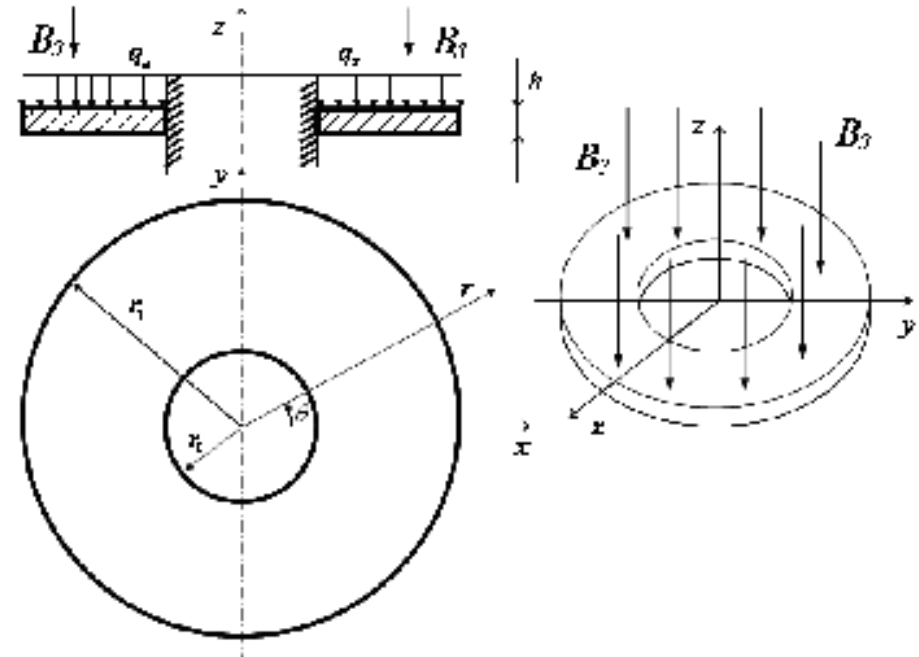

Fig.2 Schematic diagram of magnetorheological elastomer annular plate

Consider the annular plate, which is shown in Fig.2, which is composed of a magneto rheological elastic system, which is placed in a strong magnetic field, and is subjected to the action of mechanical load $q_{z}$. The basic equation is a set of equations described by the Eq.(3). Considering the Eq.(2), 
Eq.(4), Eq.(9), introducing $m=\rho \alpha$, using the Newmark stable finite difference formula, the linearized equations can be obtained.

$$
\begin{aligned}
& \frac{\mathrm{d} u^{(k+1)}}{\mathrm{d} m}=\frac{\mu_{2} \mu_{0}\left(1+x_{m}\right)^{2} h_{0}^{3}(1-v)}{\rho \phi x_{m}^{2} h}\left[\frac{1}{\left(B_{3}^{(k)}\right)^{2}} N_{1}^{(k+1)}+2 \frac{1}{B_{3}^{(k)}} \frac{1}{B_{3}^{(k+1)}} N_{1}^{(k)}-2 \frac{1}{\left(B_{3}^{(k)}\right)^{2}} N_{1}^{(k)}\right]- \\
& \frac{v}{m} u^{(k+1)}+\frac{1}{\rho}\left[-\theta_{1}^{(k)} \theta_{1}^{(k+1)}+0.5\left(\theta_{1}^{(k)}\right)^{2}\right] \\
& \frac{\mathrm{d} w^{(k+1)}}{\mathrm{d} m}=-\frac{1}{\rho} \theta_{1}^{(k+1)} \\
& \frac{\mathrm{d} \theta_{1}^{(k+1)}}{\mathrm{d} m}=\frac{12 \mu_{2} \mu_{0}\left(1+x_{m}\right)^{2} h_{0}^{3}(1-v)}{\rho \phi x_{m}^{2} h^{3}}\left[\frac{1}{\left(B_{3}^{(k)}\right)^{2}} M_{1}^{(k+1)}+2 \frac{1}{B_{3}^{(k)}} \frac{1}{B_{3}^{(k+1)}} M_{1}^{(k)}-2 \frac{1}{\left(B_{3}^{(k)}\right)^{2}} M_{1}^{(k)}\right]-\frac{v}{m} \theta_{1}^{(k+1)} \\
& \frac{\mathrm{d} N_{1}^{(k+1)}}{\mathrm{d} m}=\frac{1}{m}\left\{(v-1) N_{1}^{(k+1)}+\frac{2 \phi(1+v) x_{m}^{2} h}{\mu_{2} \mu_{0}\left(1+x_{m}\right)^{2} h_{0}^{3} r}\left[-\sigma \mu\left(B_{3}^{(k)} u^{(k)} E_{2}^{(k+1)}+B_{3}^{(k)} u^{(k+1)} E_{2}^{(k)}+\right.\right.\right. \\
& \left.B_{3}^{(k+1)} u^{(k)} E_{2}^{(k)}-2 B_{3}^{(k)} u^{(k)} E_{2}^{(k)}\right)+\sigma \mu\left(\left(B_{3}^{(k)}\right)^{2} u^{(k)} \frac{2\left(u^{(t+\Delta t)}\right)^{(k+1)}}{\Delta t}-\left(B_{3}^{(k)}\right)^{2} u^{(k)} \frac{2\left(u^{(t)}\right)^{(k)}}{\Delta t}-\right. \\
& \left(B_{3}^{(k)}\right)^{2} u^{(k)}\left(\dot{u}^{(t)}\right)^{(k)}+\left(B_{3}^{(k)}\right)^{2} u^{(k+1)}\left(\dot{u}^{(t+\Delta t)}\right)^{(k)}+2 B_{3}^{(k)} B_{3}^{(k+1)} u^{(k)}\left(\dot{u}^{(t+\Delta t)}\right)^{(k)}- \\
& \left.\left.3\left(B_{3}^{(k)}\right)^{2} u^{(k)}\left(\dot{u}^{(t+\Delta t)}\right)^{(k)}\right)\right]+\frac{\phi(1+v) x_{m}^{2} h}{\mu_{2} \mu_{0}\left(1+x_{m}\right)^{2} h_{0}^{3} r}\left[\frac{\mu_{2} \mu_{0}\left(1+x_{m}\right)^{2} h_{0}^{3}(1-v)}{\phi x_{m}^{2} h} N_{1}^{(k+1)}-\right. \\
& \frac{v}{r}\left(B_{3}^{(k)} u^{(k+1)} B_{3}^{(k)}+2 B_{3}^{(k)} B_{3}^{(k+1)} u^{(k)}-2\left(B_{3}^{(k)}\right)^{2} u^{(k)}\right)-0.5\left(2\left(B_{3}^{(k)}\right)^{2} \theta_{1}^{(k+1)} \theta_{1}^{(k)}+\right. \\
& \left.\left.\left.2 B_{3}^{(k)} B_{3}^{(k+1)}\left(\theta_{1}^{(k)}\right)^{2}-3\left(B_{3}^{(k)}\right)^{2}\left(\theta_{1}^{(k)}\right)^{2}\right)\right]\right\}+h\left(\frac{4\left(u^{(t+\Delta t)}\right)^{(k+1)}}{(\Delta t)^{2}}-\frac{4\left(u^{(t)}\right)^{(k)}}{(\Delta t)^{2}}-\right. \\
& \left.\frac{4\left(\dot{u}^{(t)}\right)^{(k)}}{\Delta t}-\left(\ddot{u}^{(t)}\right)^{(k)}\right) \\
& \frac{\mathrm{d} Q_{1}^{(k+1)}}{\mathrm{d} m}=-\frac{1}{m} Q_{1}^{(k+1)}-\frac{4 \pi R_{0}^{3}\left(\mu_{2}-\mu_{1}\right) \mu_{1}}{\rho \mu_{0}^{2}\left(\mu_{2}+2 \mu_{1}\right)\left(1+x_{m}\right)^{2}}\left\{-\sigma \mu\left(B_{3}^{(k+1)} E_{2}^{(k)}+B_{3}^{(k)} E_{2}^{(k+1)}-\right.\right.
\end{aligned}
$$$$
B_{3}^{(k)} E_{2}^{(k)}+\sigma \mu\left[\left(B_{3}^{(k)}\right)^{2}\left(\frac{2\left(u^{(t+\Delta t)}\right)^{(k+1)}}{\Delta t}-\frac{2\left(u^{(t)}\right)^{(k)}}{\Delta t}-\left(\dot{u}^{(t)}\right)^{(k)}\right)+\right.
$$$$
\left.\left.2 B_{3}^{(k)} B_{3}^{(k+1)}\left(u^{(t+\Delta t)}\right)^{(k)}-2\left(B_{3}^{(k)}\right)^{2}\left(u^{t+\Delta t}\right)^{(k)}\right]\right\}
$$$$
h\left(\frac{4\left(w^{(t+\Delta t)}\right)^{(k+1)}-4\left(w^{(t)}\right)^{(k)}}{(\Delta t)^{2}}-\frac{4\left(w^{(t)}\right)^{(k)}}{\Delta t}-\left(w^{(t)}\right)^{(k)}\right)
$$

$$
\begin{aligned}
\frac{\mathrm{d} M_{1}^{(k+1)}}{\mathrm{d} m}= & \frac{1}{m}\left\{(v-1) M_{1}^{(k+1)}+\frac{\phi(1+v) x_{m}^{2} h^{3}}{6 r \mu_{2} \mu_{0}\left(1+x_{m}\right)^{2} h_{0}^{3}}\left[-\sigma \mu\left(B_{3}^{(k)} \theta_{1}^{(k)} E_{2}^{(k+1)}+B_{3}^{(k)} \theta_{1}^{(k+1)} E_{2}^{(k)}\right.\right.\right. \\
& \left.B_{3}^{(k+1)} \theta_{1}^{(k)} E_{2}^{(k)}-2 B_{3}^{(k)} \theta_{1}^{(k)} E_{2}^{(k)}\right)+\sigma \mu\left(\left(B_{3}^{(k)}\right)^{2} \theta_{1}^{(k)} \frac{2\left(u^{(t+\Delta t)}\right)^{(k+1)}}{\Delta t}-\right. \\
& \left(B_{3}^{(k)}\right)^{2} \theta_{1}^{(k)} \frac{2\left(u^{(t)}\right)^{(k)}}{\Delta t}-\left(B_{3}^{(k)}\right)^{2} \theta_{1}^{(k)}\left(\dot{u}^{(t)}\right)^{(k)}+\left(B_{3}^{(k)}\right)^{2} \theta_{1}^{(k+1)}\left(\dot{u}^{(t+\Delta t)}\right)^{(k)}+ \\
& 2 B_{3}^{(k)} B_{3}^{(k+1)} \theta_{1}^{(k)}\left(\dot{u}^{(t+\Delta t)}\right)^{(k)}-3\left(B_{3}^{(k)}\right)^{2} \theta_{1}^{(k)}\left(\dot{u}^{(t+\Delta t)}\right)^{(k)} \\
& \frac{\phi(1+v) x_{m}^{2} h^{3}}{12 r \mu_{2} \mu_{0}\left(1+x_{m}\right)^{2} h_{0}^{3}}\left[\frac{12 \mu_{2} \mu_{0}\left(1+x_{m}\right)^{2} h_{0}^{3}(1-v)}{\phi x_{m}^{2} h^{3}} M_{1}^{(k+1)}-\frac{v}{r}\left(B_{3}^{(k)} \theta_{1}^{(k+1)} B_{3}^{(k)}+\right.\right.
\end{aligned}
$$




$$
\begin{gathered}
\left.\left.\left.2 B_{3}^{(k)} B_{3}^{(k+1)} \theta_{1}^{(k)}-2\left(B_{3}^{(k)}\right)^{2} \theta_{1}^{(k)}\right)\right]\right\}+\frac{1}{\rho}\left(N_{1}^{(k+1)} \theta_{1}^{(k)}+N_{1}^{(k)} \theta_{1}^{(k+1)}-N_{1}^{(k)} \theta_{1}^{(k)}\right)+ \\
\frac{1}{\rho} Q_{1}^{(k+1)}+\frac{h^{3}}{12}\left(\frac{4\left(\theta_{1}^{(t+\Delta t)}\right)^{(k+1)}-4\left(\theta_{1}^{(t)}\right)^{(k)}}{(\Delta t)^{2}}-\left(\frac{4\left(\dot{\theta}_{1}^{(t)}\right)^{(k)}}{\Delta t}-\left(\ddot{\theta}_{1}^{(t)}\right)^{(k)}\right)\right) \\
\frac{\mathrm{d} B_{3}^{(k+1)}}{\mathrm{d} m}=-\frac{1}{\rho} \sigma \mu E_{2}^{(k+1)}+\frac{1}{\rho} \sigma \mu\left[-\left(\dot{u}^{(t+\Delta t)}\right)^{(k)} B_{3}^{(k)}+\right. \\
\left(\frac{2\left(u^{(t+\Delta t)}\right)^{(k+1)}}{\Delta t}-\frac{\left(u^{(t)}\right)^{(k)}}{\Delta t}-\left(\dot{u}^{(t)}\right)^{(k)}\right) B_{3}^{(k)}+\left(\dot{u}^{(t+\Delta t)}\right)^{(k)} B_{3}^{(k+1)} \\
\frac{\mathrm{d} E_{2}^{(k+1)}}{\mathrm{d} m}=\frac{1}{\rho}\left(-\frac{\left(B_{3}^{(t+\Delta t)}\right)^{(k+1)}}{\Delta t}+\frac{2\left(B_{3}^{(t)}\right)^{(k)}}{\Delta t}+\left(\dot{B}_{3}^{(t)}\right)^{(k)}\right)-\frac{E_{2}^{(k+1)}}{m}
\end{gathered}
$$

Considering the geometrical dimensions of the annular thin plate as shown in Fig.2 as follows: thickness $h=2 \times 10^{-3} \mathrm{~m}$, inner radius $r_{0}=0.5 \mathrm{~m}$, outer radius $r_{1}=1.0 \mathrm{~m}$, Poisson ratio $v=0.49$, mass density $\rho=1000 \mathrm{~kg} / \mathrm{m}^{3}$, load intensity $q_{z}=-5 \mathrm{~Pa}$, the characteristic parameters of magnetic field ${ }^{[10]}: \sigma=1.0 \times 10^{-2}(\Omega \cdot \mathrm{m})^{-1}, \mu_{1}=1000 \mathrm{H} / \mathrm{m}, \mu_{0}=1.256 \times 10^{-6} \mathrm{H} / \mathrm{m}, \mu_{2}=1.0 \mathrm{H} / \mathrm{m}, x_{m}=1000$, $\mu=3.34 \mathrm{H} / \mathrm{m}, h_{0}=1, \phi=0.3, R_{0}=1.0 \times 10^{-6} \mathrm{~m}$.

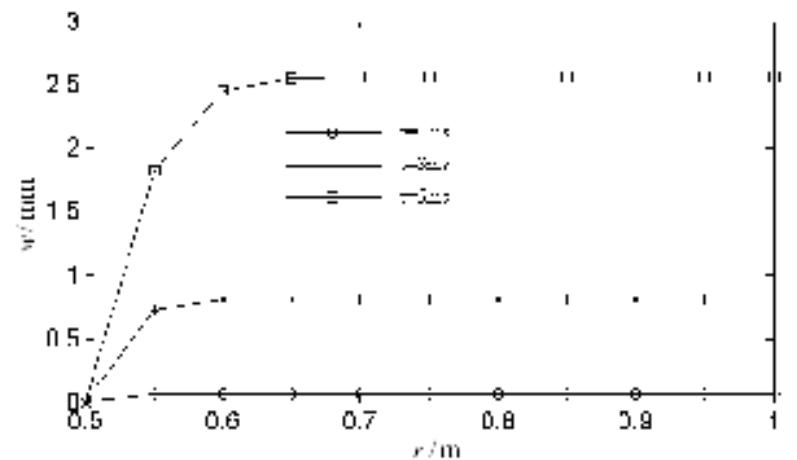

Fig.3 relationship between deflection of annular plate and the time

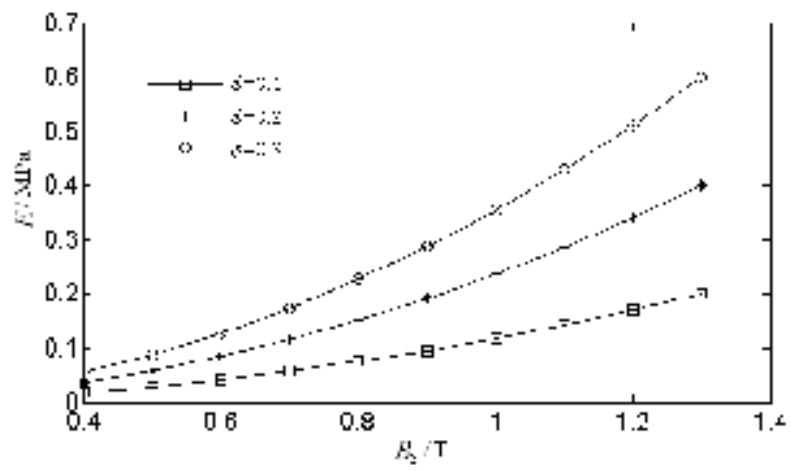

Fig.4 relationship between the elastic modulus of annular plate and the magnetic induction intensity

Fig. 3 shows the deflection of the magneto rheological elastic annular plate at $t=1 \mathrm{~ms}, t=3 \mathrm{~ms}$, $t=5 \mathrm{~ms}$ moment, it can be seen that the same position of the annular thin plate increases with the increase of time. At the same time, the deflection of the annular plate becomes larger and larger from the inside out, and the deflection of the inner ring changes rapidly, and the deflection of the outer side tends to be gentle. Fig. 4 shows that the elastic modulus of the magnetorheological elastomer annular plate varies with the applied magnetic field when the particle volume concentration ratio is $\phi=0.1$, $\phi=0.2, \phi=0.3$ respectively, the law is consistent with the experimental results of [11]. It can be seen that in the case of constant particle volume concentration, the change of the external magnetic field can cause the change of the elastic modulus of the annular plate, when the external magnetic field is constant, the volume fraction of particles is also an important factor affecting the elastic modulus of annular thin plate.

\section{Conclusions}

(1) It is possible to calculate the stress and deformation of magneto rheological elastomer components under the coupling of magnetic field and mechanical field by the magnetoelastic coupling field theory, the key is to establish the dynamic equations of the magneto rheological elastomer components under the coupling field, and it is classified as a series of differential equations, then the numerical solution of 
the problem can be obtained by the discrete orthogonal method.

(2) For a component made of magnetorheological elastomer material, the characteristics of elastic modulus with the change of magnetic induction need to be noticed. As can be seen from Figure 4, when the magnetic field increases, the elastic modulus increases rapidly, which is reflected in the increase of the bending stiffness of the component, on the other hand, the increase of the magnetic field will lead to the increase of the electromagnetic force in the surface, which will increase the deformation of the plate, the effect of both will offset some of the deformation effect. Therefore, this is something that needs attention, when the magnetic deformation effect is used for the intelligent control of the magneto rheological elastomer components

\section{Acknowledgements}

This work was financially supported by the Hebei Natural Science Foundation (A2015203086).

\section{References}

[1] X.L. Gong, H.X. Deng, J.F. Li, et al: Journal of University of Science and Technology of China Vol. 37 (2007), p. 1193-1203(in Chinese)

[2] C.J. Kang, X.L. Gong, X.M.Chen, et al: Journal of Vibration and Shock Vol. 31 (2012), p. 28-31. (in Chinese)

[3] A. Dorfmann, I. A. Brigadnov: Computational Materials Science Vol. 29 (2004), p. 270-282.

[4] L.C. Davis: JournaI of Applied Physics Vol. 85 (1999) , p. 3348-3351.

[5] R. Fosdick, H. Tang: J. Elasticity Vol. 88 (2007), p. 255-297.

[6] M. Ottenio, M. Destrade, R.W. Ogden: J. Elasticity Vol. 90 (2008) , p. 19-42.

[7] X.Z. Bai, Z.G. Tian: Plate and shell magnetic elasticity base (Science Press, Beijing 2006) (in Chinese)

[8] M. Yu, X.R. Yan, Z.L. Mao: Materials Review Vol. 21 (2007), p. 103-106. (in Chinese)

[9] M.Y.Shi, L.Z.Guo:The Computation Basis of Science and Engineering (Press of Tsinghua university, Beijing 1999) (in Chinese)

[10] Y.Shen, M.F.Golnaraghi, G.R.Heppler: Journal of Intelligent Material Systems and Structures Vol.15 (2004), p.27-34.

[11] M.R. Jolly, D.J. Carlson, B.C. Munnoz: Smart Mater Struct Vol. 5 (1996) , p.607 